\title{
Uterine Arteriovenous Malformation: A Rare Cause of Secondary Postpartum Hemorrhage
}

\author{
Caroline Chiew Ping Tan ${ }^{\mathrm{a}, \mathrm{c}}$, Manisha Mathur ${ }^{\mathrm{a}}$, \\ Richard Hoau Gong Lo ${ }^{b}$
}

\begin{abstract}
Ruptured uterine arteriovenous malformation (AVM) such as uterine artery pseudoaneurysm is an extremely rare cause of secondary postpartum hemorrhage ( $\mathrm{PPH})$, thus causing a diagnostic and therapeutic dilemma for the managing obstetricians. We describe a case of a 40-year-old lady who presented with recurrent intractable secondary PPH unresponsive to conventional treatment following an emergency lower segment cesarean section (LSCS). This was her second LSCS for a failed trial of vaginal birth after cesarean section (VBAC). Subsequently left uterine artery extravasation was identified on pelvic angiogram and selective embolization was done with successful treatment of her PPH. To date, there have been only case reports or case series available on literature due to its rarity of the diagnosis. We wish to raise awareness of this rare diagnosis in patients with risk factors as a cause of recurrent PPH not responding to medical management. We also wish to share our experience with the successful use of selective uterine artery embolization for the management of this condition which resulted in avoidance of hysterectomy and its associated morbidity.
\end{abstract}

Keywords: Uterine arteriovenous malformation; Postpartum hemorrhage; Cesarean section; Pelvic angiogram; Uterine artery embolization; Uterine artery pseudoaneurysm

\section{Introduction}

Uterine arteriovenous malformation (AVM) is an abnormal connection between vessels and can be found in any part of the body. It can be congenital or acquired. In obstetrics and gynecology, an AVM may be acquired either following treatment of miscarriage, termination of pregnancy, or following a cesar-

Manuscript accepted for publication April 18, 2017

aDepartment of Obstetrics \& Gynaecology, KK Women's and Children's Hospital, Singapore

${ }^{b}$ Department of Diagnostic Radiology, Singapore General Hospital, Singapore ${ }^{\mathrm{c} C}$ Corresponding Author: Caroline Chiew Ping Tan, Department of Obstetrics \& Gynaecology, KK Women's and Children's Hospital, 100 Bukit Timah Road, Singapore 229899, Singapore. Email: caroline.tan@mohh.com.sg

doi: https://doi.org/10.14740/jmc2804w ean section. When ruptured, AVM can lead to potentially lifethreatening intractable hemorrhage. It is one of the extremely rare causes of $\mathrm{PPH}$ and presents great difficulty in recognition and initiation of correct treatment.

\section{Case Report}

Our patient was a 40-year-old para 2 with a history of lower segment cesarean section (LSCS) in her first pregnancy. She had no other surgical or medical issues of note. Her current pregnancy had been uneventful with a planned elective repeat LSCS at 39 weeks. She presented in spontaneous labor prior to her scheduled surgery and opted for a trial of vaginal birth after cesarean section (VBAC). However, there was failure to progress beyond $6 \mathrm{~cm}$ dilatation and she underwent an emergency LSCS. The procedure was complicated due to intra-abdominal adhesions resulting in injury to urinary bladder which was repaired in layers. The blood loss during the procedure was average. The patient made good recovery and was discharged home on third day post-procedure with the indwelling urinary catheter in situ. The catheter was removed 1 week post-procedure and patient showed complete recovery. However, she presented to the emergency department on day 25 postnatal with significant secondary PPH associated with an episode of hypovolemic shock. Following resuscitation, she underwent an examination under anesthesia (EUA). Suction evacuation of the uterus was done and blood clots along with a small amount of retained products of conception (RPOC) were obtained. The blood loss was estimated as $2.5 \mathrm{~L}$ and she received 4 units packed red cells, 2 units fresh frozen plasma, and 1 unit platelets. She was also given uterotonics such as carbetocin, prostaglandin analogues such as carboprost and misoprostol along with intravenous (IV) tranexamic acid for control of bleeding. She received broad spectrum IV antibiotics due to the possibility of endometritis. Her bleeding settled and following a period of observation she was discharged on third post-operative day. A week following her discharge, she re-presented to the emergency department of our hospital with another episode of significant bleeding. On account of ongoing bleeding, she underwent another EUA and evacuation of uterus with a possibility of proceeding to a laparotomy and a hysterectomy as a last resort. The interventional radiologist was alerted as the possibility of uterine artery embolization (UAE) was also under consideration. There was minimal curettings obtained during the evacuation of uterus which 


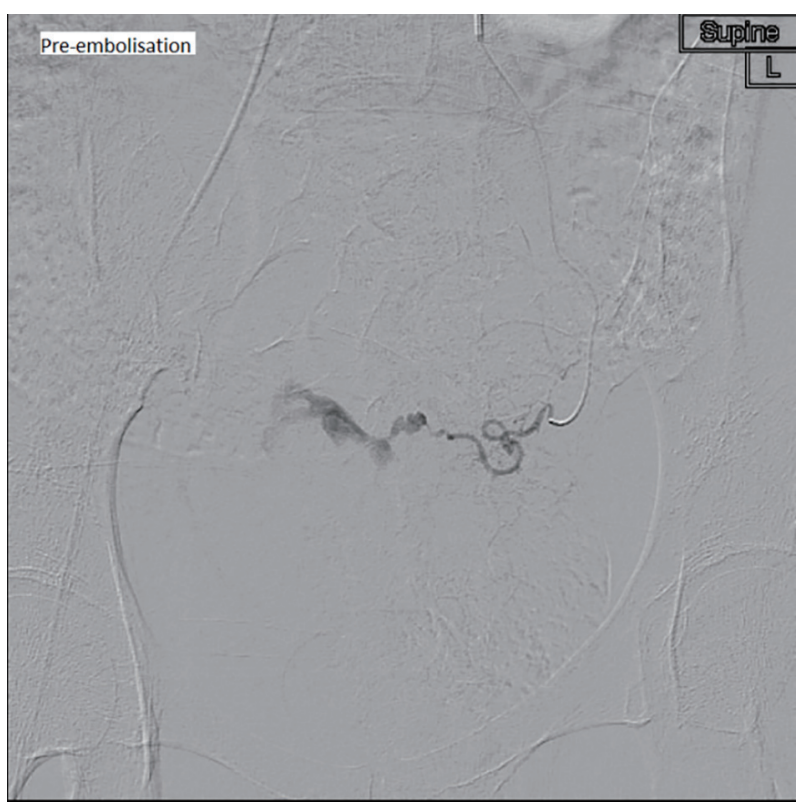

Figure 1. Superselective left uterine artery angiogram showing gross contrast extravasation/pooling medially in the pelvis pre-embolization.

was carried out under ultrasound guidance. A pelvic angiogram done showed profuse contrast extravasation and acute bleeding from the left uterine artery (Fig. 1). A super-selective embolization of left uterine artery was performed. Post-embolization angiogram showed satisfactory results with no further contrast extravasation (Fig. 2). Thereafter the patient recovered well and was discharged well after a week. Currently, 6 months postembolization, she is under follow-up for irregular periods.

\section{Discussion}

PPH is one of the most important causes of maternal mortality and morbidity [1]. According to the World Health Organization, it affects approximately $2 \%$ of all women in relation to childbirth and is the leading cause of maternal mortality in most low-income countries [2]. According to the MBRRACE (UK) report of December 2014, from the year 2009 to 2012, obstetric hemorrhage in the UK accounts for approximately $10 \%$ of all direct maternal deaths [3]. It has an overall mortality rate of 0.49 per 100,000 maternities (CI 0.29 - 0.78) and a case fatality rate for massive hemorrhage of approximately 1 per 1,200 women [3].

PPH is commonly defined as a blood loss of $500 \mathrm{~mL}$ or more within $24 \mathrm{~h}$ after birth [2]. Severe PPH is defined as a blood loss of $1,000 \mathrm{~mL}$ or more within the same time period after birth [2]. It may also be classified as primary and secondary [1]. While primary PPH occurs within the period from delivery up to $24 \mathrm{~h}$ post-delivery [4], secondary PPH occurs from the period between $24 \mathrm{~h}$ and 12 weeks postnatal [4]. The most common causes include uterine atony and genital tract trauma while the less common causes include uterine rupture, retained placental tissue, infection or maternal coagulopathy disorders [2]. Ruptured uterine AVM or pseudoaneurysm as a cause of

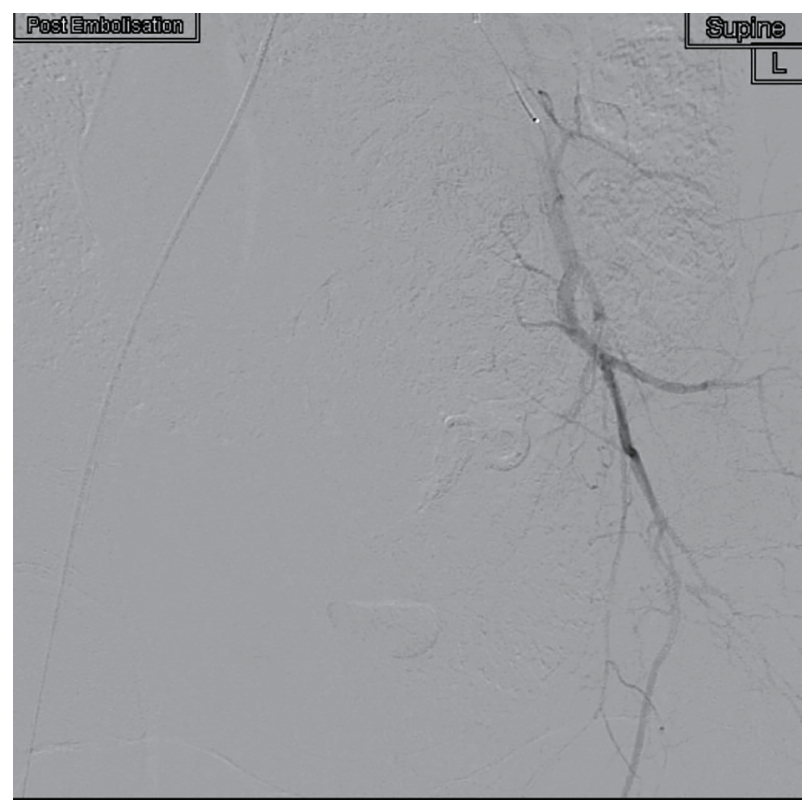

Figure 2. Post-embolization angiogram with catheter pulled back into the left IIA, showing no further contrast extravasation from the left uterine artery (uterine artery completely occluded with no opacification).

intractable secondary PPH is an extremely rare phenomenon but should be considered and looked for in intractable PPH where other common causes have been excluded [5].

Uterine AVM can be congenital or acquired, when it is also known as a "pseudoaneurysm" of the uterine artery [1]. An acquired uterine AVM is characterized as a communication between the branches of the uterine artery and the myometrial venous plexus [6]. It rarely affects nulliparous women without a history of uterine instrumentation or surgery such as evacuation of uterus, cesarean section, dilatation and curettage [6]. Other than iatrogenic causes, pathologic processes such as infection, trophoblastic disease, and malignancies involving the uterus have also been found to be associated with acquired uterine AVM [6]. In obstetric, there is another form of acquired AVM called pseudoaneurysm of the uterine arteries, which is more likely to be the cause in case of intractable secondary $\mathrm{PPH}$ [1]. It is said that trauma to the uterine arteries during LSCS is commonly found to be associated with this type of vascular abnormality [1]. The hypothesis is that it may be due to lateral extension of the lower uterine segment incision and failure to secure the apex during repair, thus leading to formation of a pseudoaneurysm [1].

Uterine AVM has traditionally been diagnosed during laparotomy or during pathological examination of the uterus after a hysterectomy [7]. The method of diagnosing uterine AVMs has traditionally been proved to be difficult [8]. Typical diagnostic procedures such as pelvic examination, hysteroscopy and gray-scale ultrasonography have not been found to be adequately effective in diagnosing this condition [8]. In recent years, Doppler ultrasound studies and focused magnetic resonance imaging (MRI) has been found to be useful in diagnosing uterine AVM [9]. It provides a non-invasive method of diagnosis of uterine AVMs $[8,9]$. MRI is mostly helpful 
where there is doubt in diagnosis on ultrasonography and there is an aversion to invasive angiography [10]. The current gold standard in diagnosing uterine AVM is by pelvic angiography which maps out the pelvic vascular anatomy [8]. It also helps to identify the bleeder and its leading "feeder" vessels whereby selective embolization can be done at the same setting as a conservative treatment option [8].

Historically, the main treatment for uterine AVMs has been hysterectomy [6]. However, embolization is the preferred treatment with a high success rate of $>90 \%$ [5], most commonly the uterine artery [1]. It also serves as an alternative option for patients seeking to preserve fertility [6] and major operative morbidity associated with a hysterectomy. However, it can be associated with complications including fever, neurological complications, puncture site hematomas, abscess, ischemic complications like vesicovaginal fistulas, endometrial atrophy, etc. [5]. The effect of embolization on future fertility is still unknown and is a subject of research [5].

In the case of our lady she had history of prior LSCS with last procedure performed approximately 4 weeks prior to her presentation with first of the recurring episodes of PPH. She had also undergone evacuation of uterus twice during the management of her PPH. We hypothesized that her uterine surgeries may have predisposed her to the formation of her pseudoaneurysm in her left uterine artery, a pathology which accounted for her presentation.

A review of literature shows that there are no known prevention measures for acquired AVM except for high clinical suspicion when a patient presents with intractable PPH with a background of recent surgical intervention, i.e. cesarean section [1]. There must also be diligent care during surgical repair, i.e. cesarean section to reduce the risk of developing iatrogenic vascular abnormalities that may cause such rare intractable hemorrhage [1].

\section{Conclusion}

Uterine AVM as a cause of PPH unresponsive to conventional treatment methods is rare but can be potentially life-threatening. In the case of an intractable PPH, there should be high index of clinical suspicion especially in the presence of risk factors such as prior surgical intervention. Multi-disciplinary care involving interventional radiology team with use of selective UAE as treatment method should be considered should suspicion of uterine AVM arise, due to its high success rate and minimally invasive nature.

\section{Conflicts of Interest}

None.

\section{References}

1. Osama Abu-Ghazza, Kevin Hayes, Edwin Chandraharan, Anna-Maria Belli. Vascular malformations in relation to obstetrics and gynaecology: diagnosis and treatment. The Obstetrician \& Gynaecologist. 2010;12:87-93.

2. WHO recommendations for the prevention and treatment of postpartum haemorrhage: 1. Postpartum hemorrhage - prevention and control. 2. Postpartum hemorrhage therapy. 3. Obstetric labor complications. 4. Guideline. I. World Health Organization. World Health Organization 2012.

3. Knight M, Kenyon S, Brocklehurst P, Neilson J, Shakespeare J, Kurinczuk JJ (Eds.). MBRRACE December 2014: Saving Lives, Improving Mothers' Care. Confidential Enquiries into Maternal Deaths and Morbidity 20092012. Oxford: National Perinatal Epidemiology Unit, University of Oxford 2014.

4. Prevention and Management of Postpartum Haemorrhage: Green-top Guideline No. 52. BJOG. 2017;124(5):e106e149.

5. Vatsla Dadhwal, Bindiya Gupta, DN Srivastava, Deepika Deka, Suneeta Mittal. Uterine Artery Pseudoaneurysm with AV Malformation: A Rare Cause of Secondary Post Partum Hemorrhage. JK Science. 2007;9(3).

6. Yoon DJ, Jones M, Taani JA, Buhimschi C, Dowell JD. A Systematic Review of Acquired Uterine Arteriovenous Malformations: Pathophysiology, Diagnosis, and Transcatheter Treatment. AJP Rep. 2016;6(1):e6-e14.

7. Hashim H, Nawawi O. Uterine arteriovenous malformation. Malays J Med Sci. 2013;20(2):76-80.

8. Kelly SM, Belli AM, Campbell S. Arteriovenous malformation of the uterus associated with secondary postpartum hemorrhage. Ultrasound Obstet Gynecol. 2003;21(6):602-605.

9. Farias MS, Santi CC, Lima AA, Teixeira SM, De Biase TC. Radiological findings of uterine arteriovenous malformation: a case report of an unusual and life-threatening cause of abnormal vaginal bleeding. Radiol Bras. 2014;47(2):122-124.

10. Hayes K. Vascular Malformations as a Cause of Postpartum Hemorrhage. Postpartum Haemorrhage 2nd Edn. 